\title{
Non-contacted Permanent Magnetic Absorbed Wall-climbing Robot for Ultrasonic Weld Inspection of Spherical Tank
}

\author{
Yulin Ding ${ }^{1}$, Zhenguo Sun ${ }^{1,2, *}$, and Qiang Chen ${ }^{1,2}$ \\ ${ }^{1}$ Department of Mechanical Engineering, Tsinghua University, Beijing, 100084, China \\ ${ }^{2}$ Yangtze Delta Region Institute of Tsinghua University, Jiaxing, 314006, China
}

\begin{abstract}
A novel wall-climbing robot for the onsite weld inspecting of spherical tank with Time of Flight Diffraction (TOFD) method has been developed, in order to liberate operators from dangerous and heavy working environment. Patented non-contacted permanent magnetic absorbed technology was adopted to realize reliable and flexible all-position moving along the weld seam on the surface of spherical tank. To ensure stable coupling for TOFD test, a surface adaptive probe holder which can provide constant contact force, have been particularly designed. Equipped with a visual sensing based weld seam tracking unit and industrial PC station, the robot could perform automatic flaw detection remotely even in the darkness environment. Onsite ultrasonic weld inspections have been carried out on a $4000 \mathrm{~m}^{3}$ spherical tank with $28 \mathrm{~mm}$ in thickness. It is verified that the robot could accomplish tasks in any position and the acquired TOFD images satisfy the requirements of engineering evaluation.
\end{abstract}

\section{Introduction}

Spherical tanks, as steel-made weldments, are widely used in petrochemical industry for the storage of gas or liquid. For a $4000 \mathrm{~m}^{3}$ spherical tank with $19.7 \mathrm{~m}$ in diameter, the total length of weld is $589.5 \mathrm{~m}$. The spherical tank usually works under high pressure and corrosive environment, and have critical requirements for weld quality. To ensure the safety and reliability, ultrasonic inspection is carried out to find out flaws such as cracks, holes and creeps in the weld joints, not only in the process of its build-up, but also periodically during its service life.

Currently, the weld inspections of spherical tank for both outside and inside are mainly carried out manually via TOFD method [1], and requires the all-around scaffolds to support the inspectors, as shown in Figure 1. However, there are many deficiencies in this operating manner, such as high labour intensity, long inspection period, and poor safety due to the large inspection area, the high inspection position and the insalubrious environment.

Three operators are need to fulfil the weld inspection, one is to operate the TOFD instrument, one is to move the probe, and the other is to spray water for reliable coupling between the detector and the surface of the weld, as shown in Figure 2. It will take up to 45 days to be completed the whole operation including the building and dismantling of the scaffolds [2]. Besides, when the wind speed reaches level four, the inspectors are forbidden to climb onto the spherical tank, which will increase the costs of time. Thus, it is of great practical significance to develop an automatic wall-climbing robot which could enable the ultrasonic weld inspection with greater safety and higher efficiency.

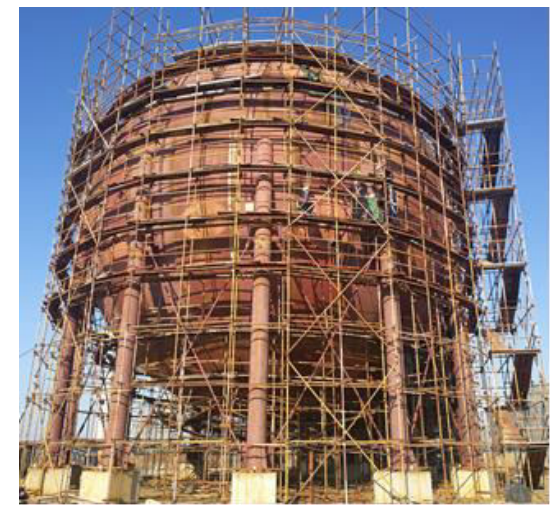

Fig. 1. Spherical tank in the construction.

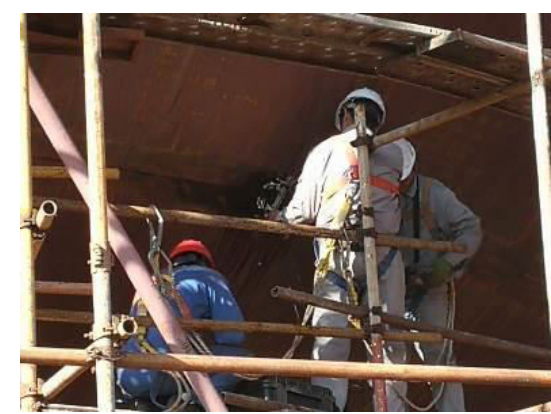

Fig. 2. Manual weld inspection of spherical tank.

According to the characteristics of the spherical tank, in order to replace manual inspection with the robotic inspection, the following challenges are faced: 
- The robot must realize both reliable adsorption and flexible movement at any position.

- In order to acquire stable TOFD images, good coupling between the wedge and work surface should be maintained during the inspection.

- In TOFD method, deviation between the centres of probes and weld seam is not allowed in excess of $5 \mathrm{~mm}$ [3]. Therefore, the robot should have a desirable automatic weld tracing performance to move along the weld seam within a deviation of $5 \mathrm{~mm}$.

Several wall-climbing robot for remote NDT inspections have been carried out. Fischer et al. proposed an automatic inspection robot that used fixed-track to determine the inspection direction and provide fixation $[4,5]$. L. P. Kalra et al. designed a climbing-robot based on tracked wheels with permanent magnets [6]. SanMillan A and Fernandez R et al. developed teleoperated climbing robots with magnetic wheels, and the robots were intended to carry ultrasonic probes for inspections $[7,8]$. However, in each case either the robot could not move to all position on the spherical tank surface, or the whole NDT equipment was not in part of the climbing robot platform.

In this paper, a novel wall-climbing robot based on non-contacted permanent magnetic absorbed technology was developed. A surface adaptive probe holder with 3 DOFs was designed to maintain the good coupling. A colour-sensitive sensor was used to estimate the weld positon for weld seam tracking. In addition, the robot was equipped with a remote control system, inspectors could operate the robot and TOFD equipment on the ground, as well as observe robot status and inspection data in real time.

\section{Non-contacted permanent magnetic absorbed wall-climbing robot}

The components of the ultrasonic weld inspection wallclimbing robot are illustrated in Figure 3. Dual drive wheels and differential steering mode was adopted in the mobile platform, and the magnetic adsorption unit was installed around the wheels. The particularly designed probe holder was mounted on the bottom plate of the mobile platform. A colour-sensitive sensor for weld tracking was installed above the centre of the probes.

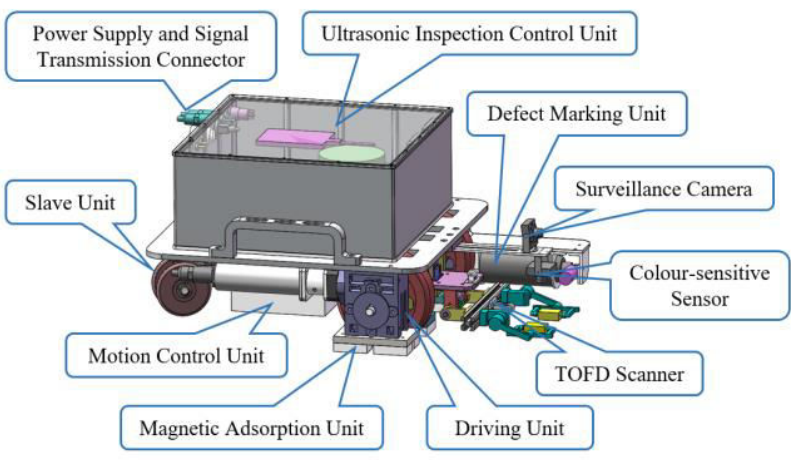

Fig. 3. Illustration of the wall-climbing robot.

\subsection{Patented technology of non-contacted permanent magnetic absorption}

Most of reported wall climbing robots with permanent magnetic adsorption method were using magnetic wheel or magnetic caterpillar technologies (as shown in Figure 4) $[9,10]$. But their load capacity, moving flexibility and surface adaptive ability cannot reach the requirements of mobile platform working on the complicated curved surface of hydraulic turbine blades. It can be found that reliable absorption and flexible moving are the paradox of static and dynamic $[11,12]$. Since magnetic wheel or magnetic caterpillar plays the role of absorption and driving together, it is very difficult to reach different requirements of both sides.
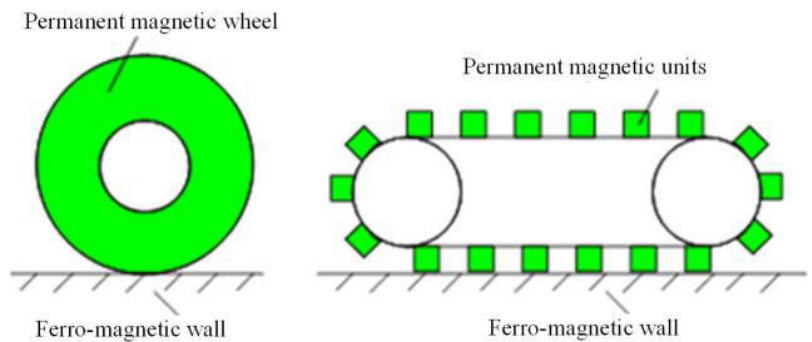

Fig. 4. Illustration of magnetic wheel or magnetic caterpillar technologies.

Hence, a novel patent technology named noncontacted magnetic adsorbed scheme was proposed (as shown in Figure 5) to overcome the shortcoming of magnetic wheel or magnetic caterpillar. In this patent, the planar permanent magnet units are fixed in bottom of the robot to provide the sucking force in need, and nonmagnetic wheels are used to support the robot. There exists a 5-8 $\mathrm{mm}$ gap between the magnetic devices and the attached surface. The robot is driven by the friction force between the wheel and the adsorption surface, which is produced by the magnetic force. This scheme can not only achieve the higher magnetic energy utilization than magnetic caterpillar or magnetic wheel, but also has the flexibility of the magnetic wheel technology [13-15].

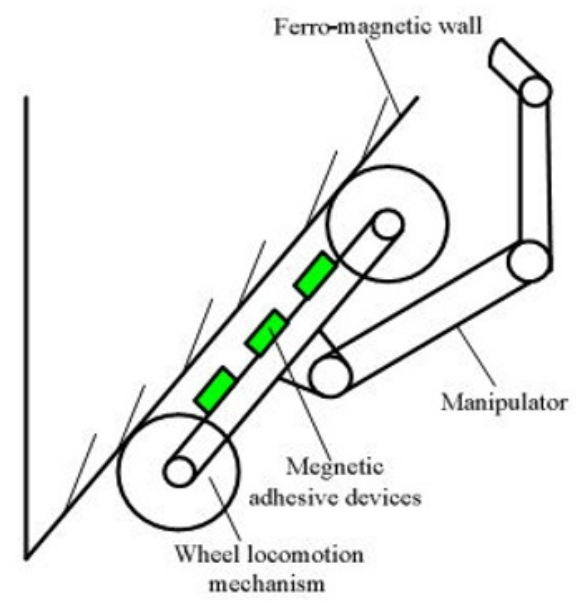

Fig. 5. Illustration of the patented non-contacted permanent magnetic absorption technology. 


\subsection{Magnetic absorption units}

Permanent magnetic adsorption unit with surface adaptability was specially designed. As shown in Figure 6 , each unit adopts six permanent magnets distributed around the driving wheel, which can minimize the distance variance between the permanent magnets and the surface of the work piece and keep the constant of the adhesion force, when the robot moves on the surfaces with different radius of curvature. As shown in Figure 7, when the inspection surface of the robot changes from the inside to the outside of the spherical tank, the distances between magnet 1, 4 and surface become larger, while the distances between magnet 2, 3 and surface become smaller, therefore the adsorption forces were adaptively adjusted to keep constant.

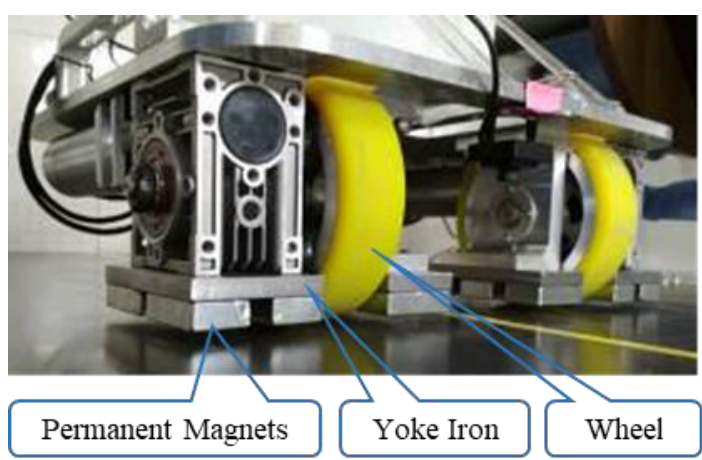

Fig. 6. Permanent magnetic adsorption units.

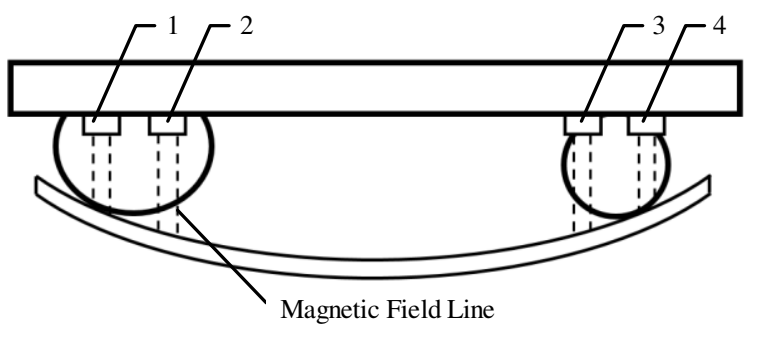

(a)

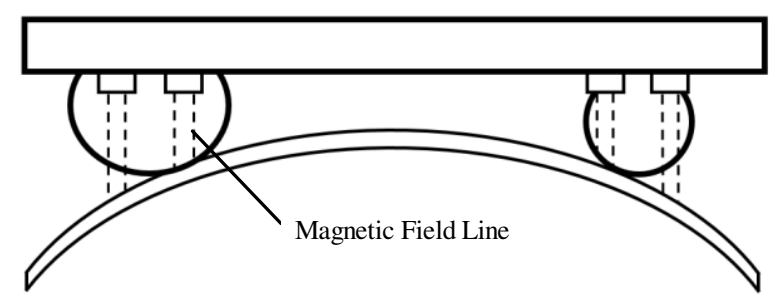

(b)

Fig. 7. Changes of adhesion force on different surfaces. (a) Inside surface, (b) Outside surface.

\subsection{Ultrasonic inspection system with TOFD method}

The ultrasonic inspection system is mainly composed of an ultrasonic inspection board, probes, wedges, probe holders and couplant supply unit. The stable and reliable signal is always desired for ultrasonic inspection to evaluate the defect of weld. Since TOFD is a very sensitive technique [16-18], apart from the inherent factors like frequency, resolution, beam spread and the presence of material noise, the coupling state between the wedge and the surface work piece has a great effect on the signal acquisition [19]. For this reason, a surface adaptive probe holder with 3 DOFs for the weld inspection robot was designed. As shown in Figure 8, the probe holder consists of a mounting frame and a main frame, and a hinge connect these two frames. The mounting frame is fixed on the bottom plate of mobile platform. Two torsion spring assemblies are used to provide the forces to press the main frame against the surface. The main frame included a frame bar, two torsion spring assemblies and probe holder arms. The two torsion spring assemblies could provide suitable contact force on the surface. The probe holder arms could be rotated around the fulcrum within a small range of angles, so as to adjust the relative positon of the probes to make them keep in good contact with the working surface during inspection.

In addition, an electric pusher was used for lifting the main frame and the probes in order to cross the obstacles such as weld seam when the robot moved to the desired areas without performing inspection task. A ring was fixed on the main frame, a fixed pulley and the electric putter were installed on the mounting frame. The ends of a rope were attached to the electric putter and the ring respectively, and the rope was arranged on the fixed pulley. So the lifting and releasing motion of the main frame and the probes could be accomplished by the telescopic action of the electric pusher.

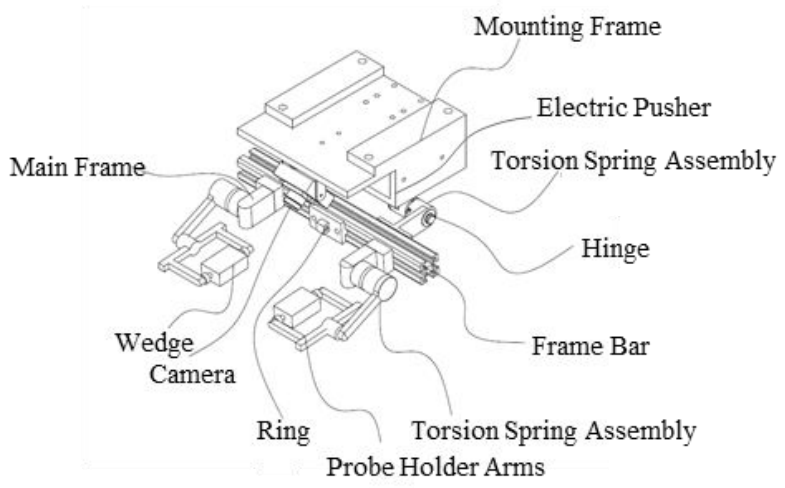

Fig. 8. Structure of probe holder.

\subsection{Client/server based control system}

In order to perform automatic flaw detection remotely, the control system of the wall-climbing robot was developed based on client/server architecture. As shown in Figure 9, the Client applications is run on the onboard controller equipped with the climbing robot, and the Server applications is run on industrial PC on the ground. 
Client/Robotic Climber

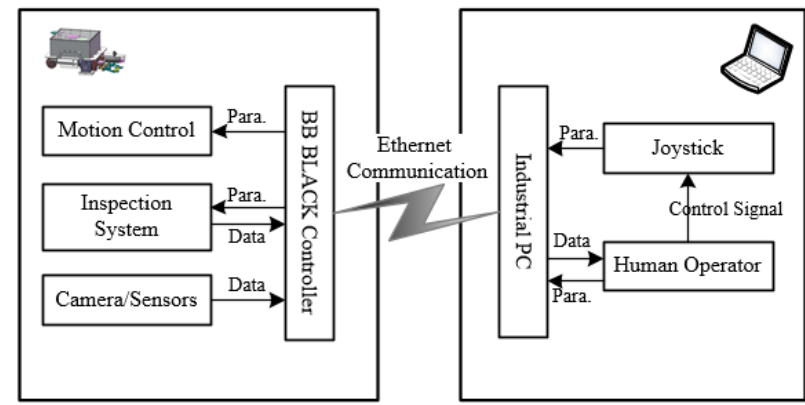

Fig. 9. Client/server based control system.

The main tasks of the server applications are to control the motion of the robot, to configure the inspection parameters and to monitor the robot status and inspection data. The monitoring station is running on an industrial PC with Intel J1900 Celeron quad-core processor and $2 \mathrm{G}$ of memory. The sending of the control parameters and the receiving of the robot status as well as TOFD inspection data are realized by Ethernet communication. A graphical user interface (as shown in Figure 5) for setting the inspection parameters, displaying and analysing the inspection data was developed. A joystick is used to switch the robot control mode (autonomous and manual control), control the robot movement, lift and release the probes, and operate the wall-climbing robot.

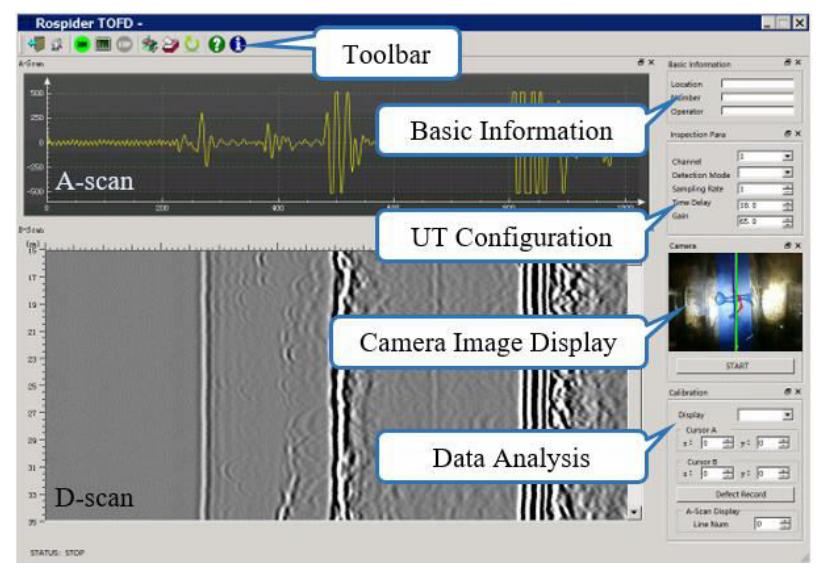

Fig. 10. Graphical user interface for TOFD inspection.

As the onboard controller, the BeagleBone Black is used to run the motion control algorithm for the robot, image processing algorithm for weld seam tracking and data acquisition algorithm for defects inspection.

Robot Operating System (ROS), which could provide a frame work for communication between robot components that is independent of hardware and runtime context, is used to implement the control system [20]. Nodes with different functions of the control system are defined and shown in Figure 11.

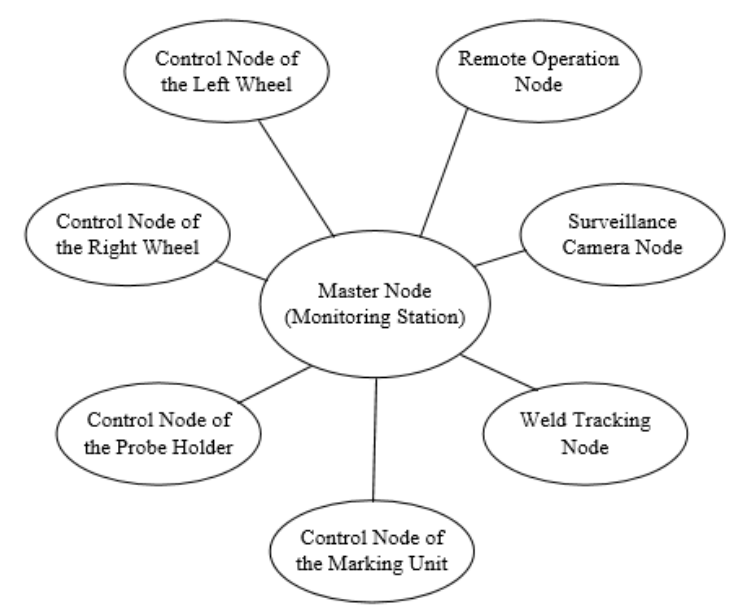

Fig. 11. Nodes with defined functions of the control system.

The robot can be operated in both autonomous and non-autonomous modes. In the manual control mode, the robot could be controlled by the operators with a joystick. For the autonomous control mode, the robot automatically follows the weld seam during inspection. In order to make the robot travel within a deviation of \pm $5 \mathrm{~mm}$ from weld seam centre, which can reach the requirement of the TOFD method, a visual image sensing based weld seam tracking unit was developed. A blue tape was painted on the surface of spherical tank to mark the position of the weld seam, and colour sensitive sensors were installed in the centreline of the probes to detect the centre of the weld seam, as shown in Figure 12.

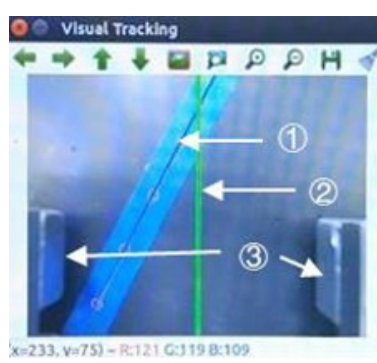

(a)

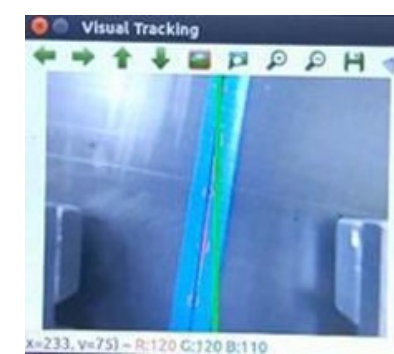

(c)

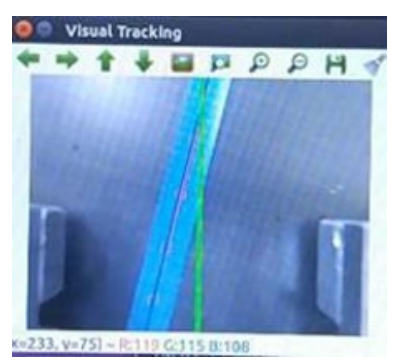

(b)

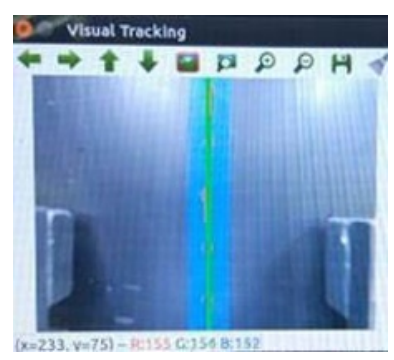

(d)
Fig. 12. Process of weld tracking.

(a) Time $=0 \mathrm{~s}$, (b) Time $=0.5 \mathrm{~s}$, (c) Time $=1 \mathrm{~s}$, (d) Time $=1.5 \mathrm{~s}$.

(1) Weld seam centre, (2) Centreline of probes, (3) Probes. 


\section{Prototype robot and on-site experiments}

The prototype of wall-climbing robot for spherical tank inspection was made and testified. The general specifications of the robot are listed in Table 1. In order to validate the effectiveness of the developed robot, onsite ultrasonic weld inspections were carried out on a $4000 \mathrm{~m}^{3}$ spherical tank with $28 \mathrm{~mm}$ in thickness, as shown in Figure 13.

Table 1. Physical specifications of the wall-climbing robot.

\begin{tabular}{cc}
\hline Item & Specification \\
\hline Mass & $25 \mathrm{~kg}$ \\
$\begin{array}{c}\text { Dimension(width, thickness, } \\
\text { height) } \\
\text { Load capacity } \\
\text { Maxim speed }\end{array}$ & $58 \times 40 \times 32 \mathrm{~cm}^{3}$ \\
Inspection speed & $10 \mathrm{~kg}$ \\
\hline
\end{tabular}

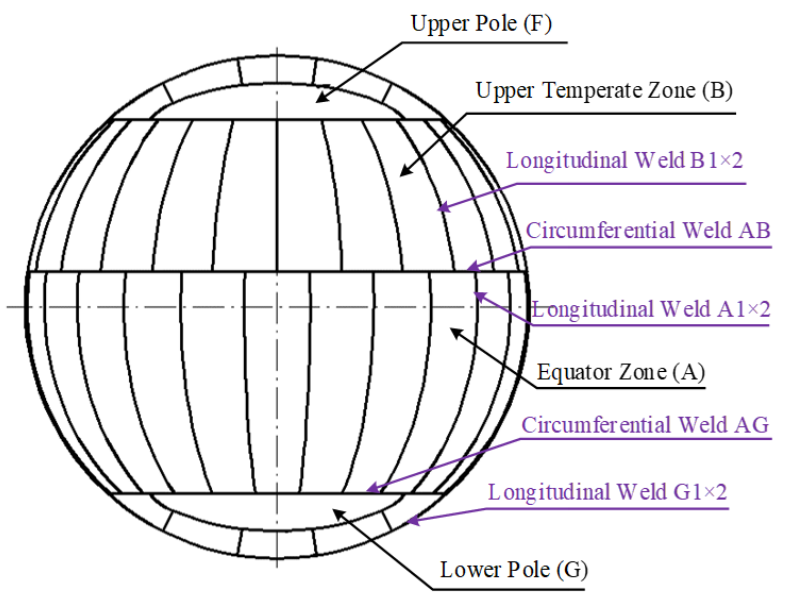

Fig. 13. Structure of $4000 \mathrm{~m}^{3}$ spherical tank.

Welds in typical locations of spherical tank (Table 2) were inspected, as shown in Figure 14. The TOFD inspection parameters are listed in Table 3. During the inspection, the robot could automatically track the weld seam and acquire satisfied TOFD images, as shown in Figure 15. A flaw was detected in longitudinal weld of upper temperate zone, as shown in Figure 15 (b). Weld inspections in the darkness environment were also conducted and qualified TOFD images could be obtained. The on-site weld inspection experiments confirm that the robot has a desirable automatic following performance for all the typical welds and can accomplish tasks in all-position, the acquired TOFD images could satisfy the requirements of engineering evaluation.
Table 2. Typical weld of spherical tank.

\begin{tabular}{lc}
\hline \multicolumn{1}{c}{ Weld Location } & Numbering \\
\hline & $\mathrm{B} 1 \times 2$ \\
\hline $\begin{array}{l}\text { Longitudinal weld in upper temperate } \\
\text { zone }\end{array}$ & $\mathrm{A} 1 \times 2$ \\
$\begin{array}{l}\text { Longitudinal weld in equator zone } \\
\text { Longitudinal weld in lower pole }\end{array}$ & $\mathrm{G} 1 \times 2$ \\
$\begin{array}{l}\text { Circumferential weld between equator } \\
\text { zone and upper temperate zone }\end{array}$ & $\mathrm{AB}$ \\
$\begin{array}{l}\text { Circumferential weld between equator } \\
\text { zone and lower pole }\end{array}$ & $\mathrm{AG}$ \\
\hline
\end{tabular}

Table 3. Parameters for TOFD inspection.

\begin{tabular}{cc}
\hline Item & Value \\
\hline Probe centre frequency & $5 \mathrm{MHz}$ \\
Beam-angle & $70^{\circ}$ \\
Probe center separation & $70 \mathrm{~mm}$ \\
\hline
\end{tabular}

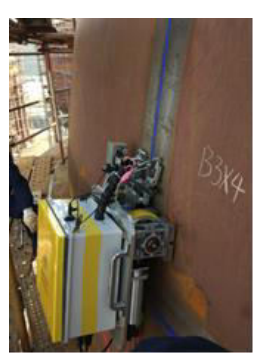

(a)

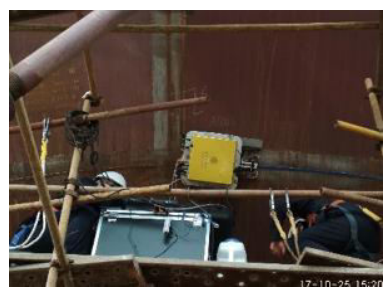

(d)

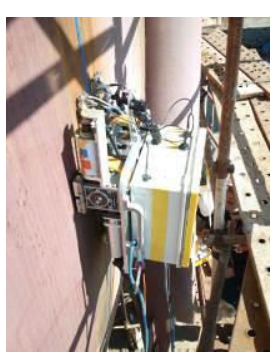

(b)

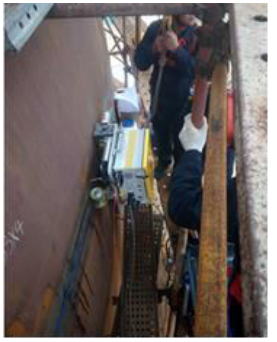

(c)

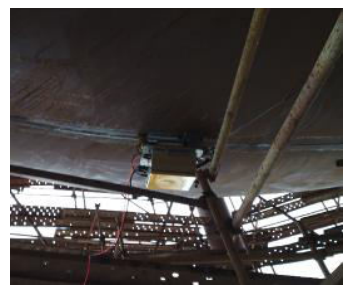

(e)
Fig. 14. On-site weld inspection of spherical tank.

(a) Longitudinal weld B1 $\times 2$, (b) Longitudinal A1 $\times 2$,

(c) Circumferential weld AB, (d) Circumferential weld AG,

(e) Longitudinal weld G1×2. 


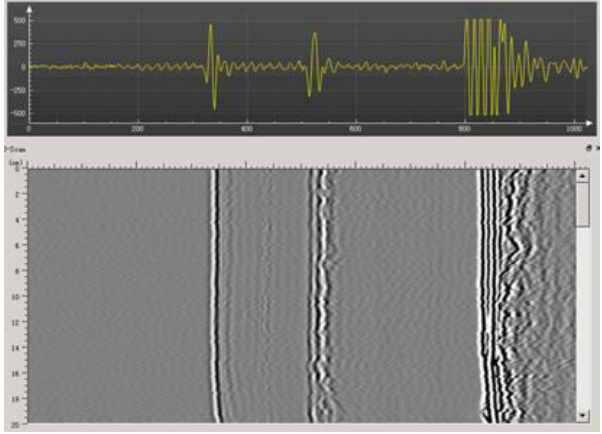

(a)

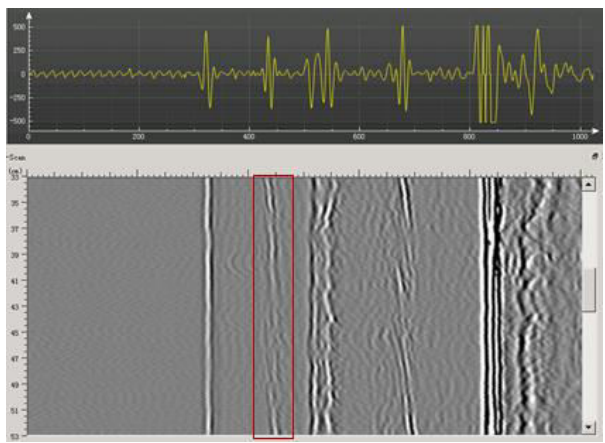

(b)

Fig. 15. TOFD images of longitudinal weld by robotic scan. (a) Weld without flaw, (b) Weld with flaw

\section{Conclusion}

A compact and portable automated ultrasonic weld inspection robot for spherical tank has been developed and testified. With the patented technology of noncontacted permanent magnetic absorption, the robot was capable of moving stably and reliably on both inside and outside surfaces of the actual spherical tank and was able to detect flaws successfully via TOFD method. For TOFD technique, a particularly designed probe holder could maintain stable and good coupling, and a visual sensing based weld seam tracking unit could keep the robot travelling within a deviation of $5 \mathrm{~mm}$ from weld seam centre during inspection. Communication by Ethernet cables allows operators to control the robot, configure inspection parameters and acquire TOFD inspection data in real time on the ground. On-site experiments conducted on the actual spherical tank show that the robot's ability to accomplish inspection tasks in all-position, even in the darkness environment. The stable and reliable TOFD images that meet the requirements of engineering evaluation could be obtained when inspection speed reaches $10 \mathrm{~mm} / \mathrm{s}$.

This research is financially supported by the National Natural Science Foundation of China (51475259) and SuzhouTsinghua Innovation Leading Action Project (2016SZ0218).

\section{References}

1. G. Nardoni, P. Nardoni, D. Nardoni, M. Feroldi, and M. Certo, METALL ITAL 11, 46 (2010).

2. Jr. J. Okamoto, Jr. V. Grassi, P. F. Santos Amaral, B. G. Miglio Pinto, D. Pipa, G. P. Pires, and M. V. Maciel Martins, J INTELL ROBOT SYST 66, 23 (2012).

3. S. Sani, M. P. Ismail, S. Mohd, N. A. Masenwat, T. S. T. Amran, M. S. M. Amin, and M. R. Ahmad, AIP Conference Proceedings (AIP, 2017).

4. W. Fischer and F. Tache and R. Siegwart, IEEE/RSJ International Conference on Intelligent Robots and Systems (2007).

5. W. Fischer, G. Caprari, R. Siegwart, I. Thommen, W. Zesch, and R. Moser, IND ROBOT 37, 244 (2010)

6. L. P. Kalra and W. Shen and J. Gu, IEEE Canadian Conference on Electrical and Computer Engineering (2006).

7. A. San-Millan, Mediterranean Conference on Control and Automation, 255 (2015).

8. R. Fernandez and E. Gonzalez and V. Feliu, IEEE Industrial Electronics Society (2010).

9. S. Han and J. Kim and H. Yi, INT J PRECIS ENG MAN 10, 143 (2009).

10. W. Shen and J. Gu and Y. Shen, Applied Bionics and Biomechanics 3, 151 (2005).

11. W. Fischer and F. Tache and R. Siegwart, Field and Service Robotics (Springer, Berlin, 2008).

12. M. Eich and T. Vogele, 19th Mediterranean Conference on. IEEE (2011).

13. Q. Chen, M. Ji, J. Wang, Z. Sun, China, ZL2002117080.0[P]. (2002-10-23).

14. J. Wang, Q. Chen, Z. Sun, China, ZL2002117079.7[P]. (2002-10-23).

15. Q. Chen, Z. Sun, W. Zhang, Z. Gui, Robotics, Automation and Mechatronics Conference on. IEEE. (2008).

16. B. Siemiatkowska, R. Chojecki, M. Wisniowski, M. Walecki, M. Wielgat, and J. Michalski, Robot Motion and Control (Springer, London, 2012).

17. W. Yi, M. Lee, J. Lee, S. Choi, and B. Lee, Key Engineering Materials (Trans Tech Publications, 2006).

18. M. Bohacik, M. Mician, R. Konar, and I. Hlavaty, Manufacturing Technology 17, 842 (2017).

19. S. Mondal and T. P. Sattar and B. Bridge, AIP Conference Proceedings (AIP, 2003).

20. M. Quigley, B. P. Gerkey, K. Conley, J. Faust, T. Foote, J. Leibs, E. Berger, R. Wheeler, and A. Y. Ng, ROS: An open-source Robot Operating System (2009). 